\title{
ANGLE-RESOLVED PHOTOEMISSION FROM SURFACES AND ADSORBATES
}

\author{
N. V. SMITH \\ Bell Laboratories, \\ Murray Hill, New Jersey 07974, U.S.A.
}

\begin{abstract}
Résumé. - En utilisant la photoémission résolue angulairement pour l'étude des surfaces, on recherche deux types d'informations fondamentales : (1) les positions des atomes à la surface et dans les espèces adsorbées; (2) la nature des états électroniques propres aux surfaces libres ainsi que celle des états impliqués dans la liaison chimique des adsorbats. Cet article présente les progrès récents obtenus dans les aspects atomique et électronique du domaine.

La détermination de l'orientation des molécules de $\mathrm{CO}$ adsorbées sur $\mathrm{Ni}$ constitue un exemple de détermination de la structure atomique par photoémission résolue angulairement.

Dans la plupart des études portant sur la zone de la bande de valence, il n'est pas facile de séparer l'information atomique et électronique, puisqu'une description complète de la structure électronique (ainsi que des spectres de la bande de valence) nécessite une connaissance des positions atomiques. On insiste sur le rôle des calculs empiriques des structures de bande à deux dimensions (relations de dispersion $E\left(k_{i}\right)$ ) à l'aide de la combinaison linéaire des orbitales atomiques (LCAO) ou des méthodes pseudo-potentielles. A titre d'exemple, on compare les relations $\mathrm{E}\left(k_{\|}\right)$mesurées par photoémission pour les états de surface sur $\mathrm{GaAs}(110)$ et les états induits par $\mathrm{Cl}$ sur $\mathrm{Si}(111)$ avec les modèles théoriques. La discussion porte sur les considérations de la parité ainsi que sur les anisotropies dans la photoémission à partir des niveaux de cœur des systèmes à deux dimensions.
\end{abstract}

\begin{abstract}
In the application of angle-resolved photoemission spectroscopy to the study of surface systems, two basic kinds of information are sought : (1) the positions of atoms in the surface and in adsorbed species; (2) the nature of the electronic states characteristic of free surfaces and those involved in the chemical binding of adsorbates. This paper surveys recent progress in both of these atomic and electronic aspects of the field. An example of an atomic-structure investigation using angle-resolved photoemission is the determination of the orientation of $\mathrm{CO}$ molecules adsorbed on $\mathrm{Ni}$. In many studies in the valence band region, the atomic and electronic information is not readily separable, since a complete description of the electronic structure (and therefore the valence band spectra) requires a knowledge of the atomic positions. The role here of semiempirical calculations of two-dimensional band structures $\left(E\left(k_{\|}\right)\right.$dispersion relations) using the Linear-Combination of Atomic Orbitals (LCAO) or pseudopotential methods is emphasized. As examples, angle-resolved photoemission measurements of the $E\left(k_{\|}\right)$relations for surface states on $\mathrm{GaAs}(110)$ and $\mathrm{Cl}$-induced states on $\mathrm{Si}(111)$ are compared with model calculations. Parity considerations and also anisotropies in core level photoemission from two-dimensional systems are discussed.
\end{abstract}

1. Introduction. - The aim of this paper is to survey, from an experimentalist's point of view, the way in which angle-resolved photoemission has extended our ability to investigate bonding at clean and adsorbate-covered surfaces. In such studies, two kinds of information are sought : atomic structure information and electronic structure information. Photoemission offers information in both these categories, although not necessarily of the same quality. Photoemission remains first and foremost a technique for the determination of the electronic structure of solids and surfaces. However, it has become such an incisive technique, particularly in its angle-resolved modification, that detailed interpretations are sensitive to atomic geometry. To put it another way, the valence electrons in a surface are probing their atomic environment, and we are probing the valence electrons. At one step removed, therefore, we are probing the atomic environment. This dual atomic/electronic capability of photoemission will be a major theme of this paper.

To mediate between experimental photoemission data and the desired surface bonding information, a theory or model of the photoemission process is required. The present situation in this regard, or at least the author's perception of it, is represented by the input/output diagram of figure 1. The ideal approach, of course, would be to interpret the experimental data in terms of a complete first-principles theory, and extract the surface bonding information as 


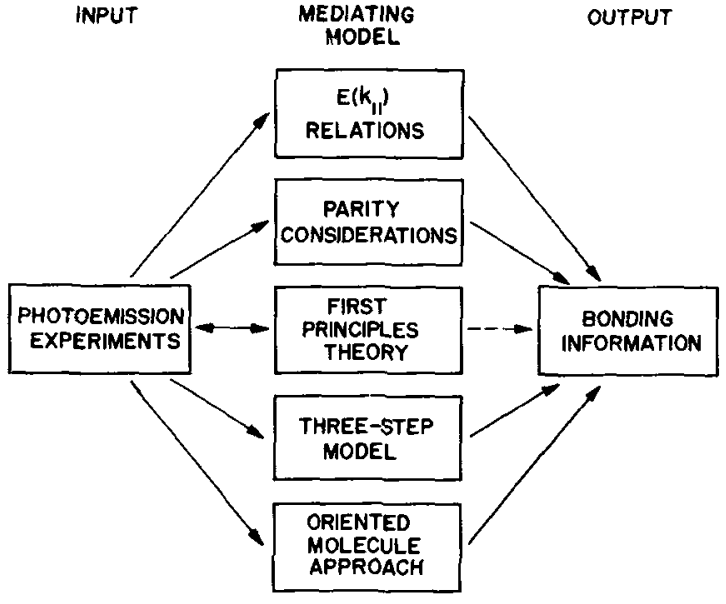

Fig. 1. - Input/output diagram showing some of the various interpretive approaches in angle-resolved photoemission.

a convenient by product. Unfortunately, the photoemission process is a complicated one [1]. Fully firstprinciples theories are still in their infancy, and their routine use in surface bonding investigations still lies in the future (hence the dashed arrow in figure 1). The activity in this important area is perhaps best represented by the double-ended arrow in figure 1 , intended to indicate that the current problem is to refine the theories and arrive at a more rigorous understanding of the physics of the photoelectric effect itself. We might appropriately refer to this as the fundamental aspect of photoemission research.

Our concern here will be with the applied rather than the fundamental aspect of photoemission. Because of the difficulties associated with the fundamental theory, the application of photoemission to the study of bonding in solids and surfaces has traditionally involved the use of simplified models, some of which are indicated in figure 1 . We will concentrate here on the new avenues of approach which have been opened up by the advent of angle-resolved photoemission. Three such avenues are listed in figure 1 under the following labels : $E\left(k_{\|}\right)$relations; parity considerations; and oriented molecule approach. These terms will be explained and discussed in subsequent sections of this paper. Also indicated in figure 1 is the three-step model. The many successes of this approximate model have been documented elsewhere [2], and will not be treated here.

This article does not pretend to be an exhaustive survey of the use of angle-resolved photoemission in surface studies. The approach will be to isolate a few key topics, with emphasis on the new avenues of interpretive approach mentioned above. These topics will be illustrated with a very limited selection of recent experimental and theoretical work. For reasons of familiarity and accessibility to the author, all the experimental results described below were obtained by the various groups who use the radiation from the Tantalus I storage ring at the University of Wisconsin
Synchrotron Radiation Centre. It should be stressed that similar experimental work has been performed by other groups at other institutions, both with and without synchrotron radiation.

2. Oriented molecule approach. - A prime example of the use of angle-resolved photoemission in a surface structural investigation is the determination of the orientation of $\mathrm{CO}$ molecules on transition metal surfaces. This kind of work was given considerable impetus by the calculations of Davenport [3]. Using a self-consistent scattered-wave $\mathrm{X} \alpha$ method, he obtained numerical results for the angular distributions of photoelectrons from oriented CO molecules. Results are shown in figure 2 for the principal valence levels $5 \sigma$,
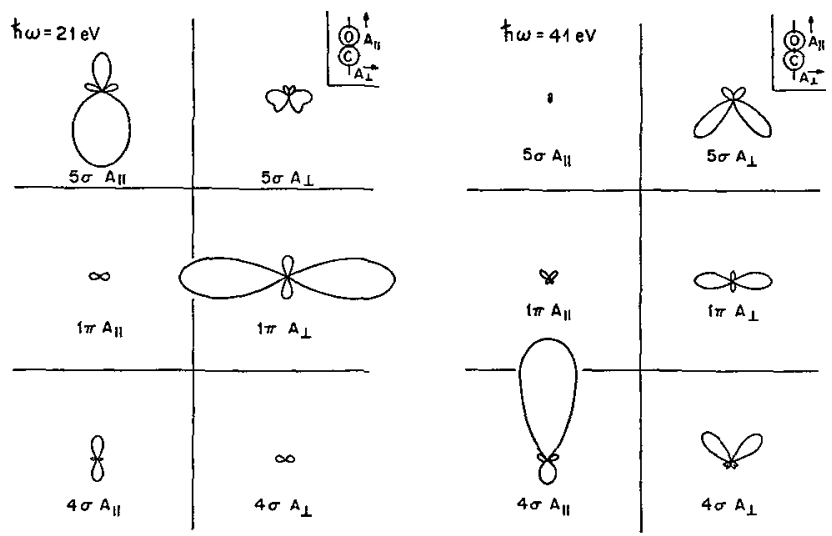

FIg. 2. - Calculated angular distributions of photoemission from the principal valence states of an oriented $\mathrm{CO}$ molecule. $A_{\|}$and $A_{\perp}$ refer to polarization directions parallel and perpendicular to the molecular axis. (Davenport, Ref. [3].)

$1 \pi$ and $4 \sigma$, at two different photon energies, and for the polarization vector $\mathbf{A}$ either parallel or perpendicular to the molecular axis. The gas phase experiment implied here cannot be performed, of course, since one would be averaging over all possible orientations. The results can be applied to adsorbed $\mathrm{CO}$ if it is assumed that the only role of the surface is to orient the molecules, and its presence can thereafter be ignored.

Some recent angle-resolved photoemission spectra taken by Allyn et al. [4] on $\mathrm{CO}$ adsorbed on $\mathrm{Ni}$ (100) for various angles of emission and polarization conditions are shown in figure 3. The $4 \sigma$ at about $-11 \mathrm{eV}$ relative to the Fermi energy $E_{\mathrm{F}}$ is indicated, and the peak labelled $P$ is a composite of the $1 \pi$ and $5 \sigma$ levels. These identifications had in fact been made earlier from comparisons between angle-integrated spectra and spectra taken on gaseous $\mathrm{CO}$ and nickel carbonyls [5]. Compared with gaseous $\mathrm{CO}$, the $5 \sigma$ level in adsorbed $\mathrm{CO}$ moves to lower energies relative to the $4 \sigma$ and $1 \pi$ levels. This immediately reveals that the $\mathrm{C}$ end of the molecule is closer to the substrate and more involved in the bonding, since the $5 \sigma$ level represents the $\mathrm{C}$ lone pair orbital. Angle-resolved studies confirm all these conclusions, but their unique contribution is in determining the precise orientation. 


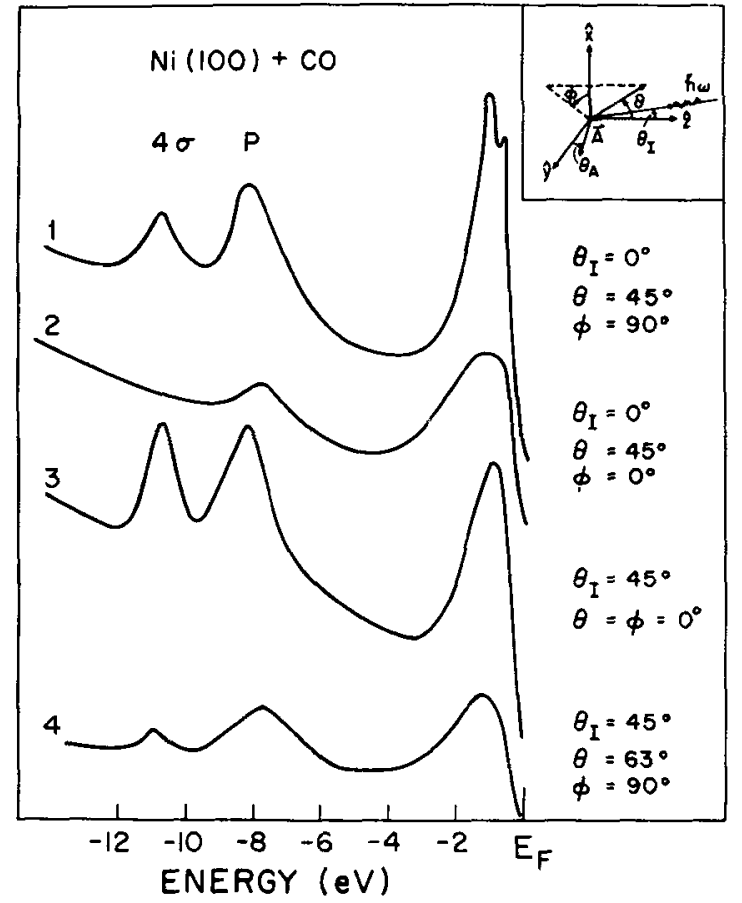

FtG. 3. - Experimental photoemission spectra for $\mathrm{CO}$ adsorbed on $\mathrm{Ni}(100)$ taken for various angles of emission and conditions of polarization. (Allyn et al., Ref. [4].)

Allyn et al. [4] conclude on the basis of spectra such as those shown in figure 3 that the $\mathrm{CO}$ molecule stands vertical on $\mathrm{Ni}$ to within an experimental accuracy of $5^{\circ}$. This conclusion is based on detailed measurements of the angular dependence of the intensity of the $4 \sigma$ peak at the photon energy $\hbar \omega=35 \mathrm{eV}$. At this photon energy the total (angle-integrated) $4 \sigma$ cross section passes through a maximum (or resonance). Davenport's calculations indicate that the resonance is associated with a final state of $\sigma$ symmetry, so that the optical selection rules are particularly clear cut. Also, the $4 \sigma$ orbital is located at the $O$ end of the molecule and is therefore least perturbed by the surface.

Davenport's scattered-wave calculations supersede a simpler model due to Gadzuk [6]. In this simpler model, it is assumed that the final state wave function in a typical optical transition is adequately represented by a plane wave $\mathrm{e}^{i \mathbf{p . r}}$ of momentum $\mathbf{p}$. In this approximation, the intensity of emission from an initial state whose wave function is $\psi(\mathbf{r})$ is given by

$$
I(\mathbf{p}) \propto(\mathbf{A} \cdot \mathbf{p})^{2}|\psi(\mathbf{p})|^{2}
$$

where $\psi(\mathbf{p})$ is the Fourier transform of $\psi(\mathbf{r})$. Since the angular dependence of an atomic orbital is the same in both real space and momentum space, we have the exciting theorem that the angular dependence of the photoemission intensity should, apart from the $(\mathbf{A} \cdot \mathbf{p})^{2}$ factor, replicate the angular dependence of the orbital itself. The results of the more sophisticated approach of Davenport are not entirely inconsistent with equation (1). Broadly speaking, the $\sigma$ states couple primarily to $A_{\|}$and have most of their emission close to the molecular axis, while the $1 \pi$ state couples primarily to $A_{\perp}$ and emits preferentially at large angles to the molecular axis. Qualitatively this is consistent with equation (1), although it is clear from figure 1 that there are instances of appreciable emission when $\mathbf{A} \cdot \mathbf{p}=0$, which is clearly contradictory to equation (1). The conclusion to be drawn here is that equation (1) is probably of use in qualitative identifications, but, because of the assumption of a plane wave final state, cannot be trusted for quantitative interpretations.

3. $E\left(\boldsymbol{k}_{\mid \mathrm{j}}\right)$ analysis : chemisorption. - We turn now to the avenue of approach referred to in the introduction as $« E\left(k_{\|}\right)$relations ». $E$ is the kinetic energy of a photoelectron, and $k_{\|}$denotes the component of the electron wave vector parallel to the surface, and is given by

$$
k_{\|}=\left(2 m E / \hbar^{2}\right)^{1 / 2} \sin \theta,
$$

where $\theta$ is the polar angle, of emission with respect to the surface normal. Monitoring $E$ as a function of $\theta$ permits mapping two-dimensional energy bands directly from experiment, as has been amply demonstrated in the case of layer compounds [7]. The same approach can be applied to certain chemisorption systems. As an example, we describe here some results obtained by Larsen et al. [8] for the saturation adsorption of $\mathrm{Cl}$ on cleaved $\mathrm{Si}$ (111). They show how, in concert with semiempirical calculations using a pseudopotential method, it is possible to arrive at a highly detailed description of the chemisorptive bond.

Some experimental photoelectron energy spectra taken on the Cl-saturated Si (111) surface at various values of $\theta$ are shown in figure 4 . The $\mathrm{Cl}$-induced peaks occur in the range -10 to $-3 \mathrm{eV}$ relative to the valence band maximum, $E_{\mathbf{v}}$. Two peaks are particularly prominent, and we digress to show that the angular dependence of the intensities of these peaks contains information on the surface structure. There are two likely possible sites for the adsorption of a $\mathrm{Cl}$ atom on the $\mathrm{Si}$ (111) surface. One is the covalent (or onefold) site in which the $\mathrm{Cl}$ atom sits directly on top of a surface $\mathrm{Si}$ atom; in this situation, the chemisorptive bond is conceived of as a covalent bond formed by the overlap of the Si dangling orbital and the $\mathrm{Cl} \mathrm{p}$ orbital ( $z$-axis taken along the surface normal). The other likely site is the ionic (or threefold) site in which the $\mathrm{Cl}$ atoms sit in the hollows formed between three neighbouring surface $\mathrm{Si}$ atoms; in this situation the $\mathrm{p}_{x, y}$ orbitals are expected to be more involved in the bonding than the $\mathrm{p}_{z}$ orbitals, although the difference should not be so pronounced as in the onefold situation.

The prominent $\mathrm{Cl}$-induced peak at about $-7.4 \mathrm{eV}$ in figure 4 has its maximum close to normal emission. 


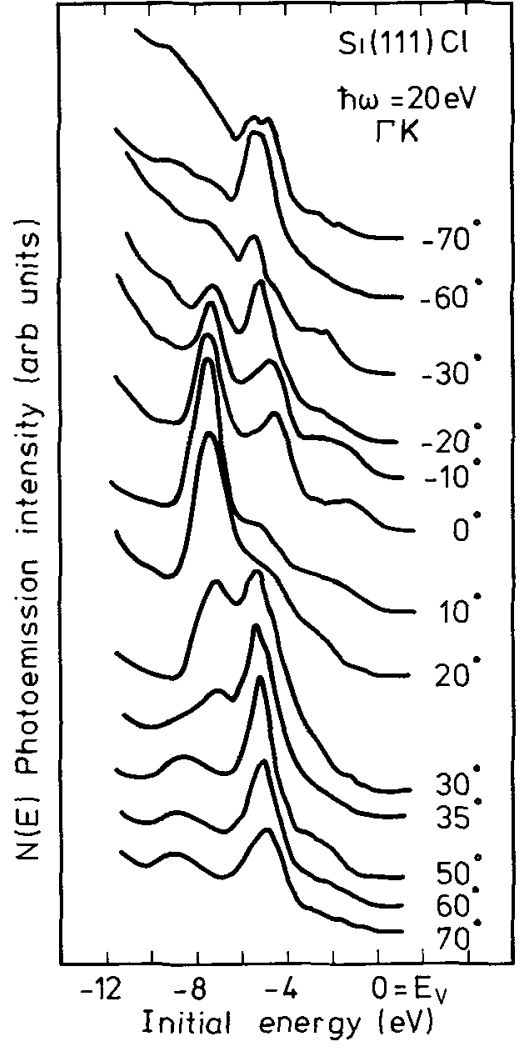

FIG. 4. - Polar angle dependence of photoemission spectra from a $\mathrm{Si}(111)$ surface covered to saturation with Cl. (Larsen et al., Ref. [8].)

According to the approximate theorem of equation (1) this is consistent with $\mathrm{p}_{z}$ symmetry. Likewise, the other prominent peak, in the -6 to $-4 \mathrm{eV}$ range, appears preferentially in the higher $\theta$ range consistent with $\mathrm{p}_{x, y}$ symmetry. These results, details of which are described elsewhere [8], favor the covalent site, in agreement with previous assignments [9].

As indicated above, variations of peak positions with angle can be reduced immediately to $E\left(k_{\|}\right)$ relations. Results for the $\Gamma \mathrm{KM}$ and $\Gamma \mathrm{M} \Gamma$ azimuths at various photon energies are summarized on the composite two-dimensional band structure of figure $5 b$. Cross hatched areas represent the projection of the bulk Si band structure onto the surface Brillouin zone. The curves in figure $5 b$ labelled $\mathrm{b}, \pi, \sigma$ and $\mathrm{s}$ represent the experimentally determined $\mathrm{Cl}$-induced bands.

Figure $5 a$ shows corresponding results of pseudopotential calculations of the surface band structure for $\mathrm{Cl}$ atoms in the covalent site. There is a strong resemblance to the experimental results of figure $5 b$. The $\sigma$ band, derived from the $\mathrm{Cl} \mathrm{p}_{z}$ orbitals, and occurring at $-7.4 \mathrm{eV}$ is rather flat in both theory and experiment. The $\pi$ band, based on the $\mathrm{Cl} \mathrm{p}_{x, y}$ orbitals, displays appreciable dispersion and has its maximum at the zone center $\Gamma$. The $\mathrm{b}$ and $\mathrm{s}$ levels seen experimentally also have their counterparts in the theoretical calculations. While overall agreement is good, there are some minor discrepancies in energy locations. For example, the $\pi-\sigma$ gap is smaller, and the s level occurs higher in energy, in experiment than in theory. These slight difficulties are attributed to the fact that the $\mathrm{Cl}$ pseudopotential in the calculations is somewhat too strong.

Apart from these minor discrepancies, the major conclusion is that the description of the chemisorptive bond given by the pseudopotential calculations is essentially correct. Further insight is then obtained by examining the details of the pseudopotential model. Pseudopotential charge densities for the four kinds of $\mathrm{Cl}$-induced states observed experimentally are shown in figure 6. The peak at $-7.4 \mathrm{eV}$ designated $\sigma$ in figures 5 and 6 is clearly seen to be strongly $\mathrm{Cl} \mathrm{p}_{z}$-like, whereas the $\pi$ peak in the -5 to $-6 \mathrm{eV}$ range is clearly $\mathrm{p}_{x, y}$ like. These calculations therefore reconfirm the original conjecture that the chemisorptive bonding proceeds primarily through a $\sigma$-like covalent bond between the $\mathrm{Cl} \mathrm{p}_{z}$ orbitals and the Si dangling bonds.
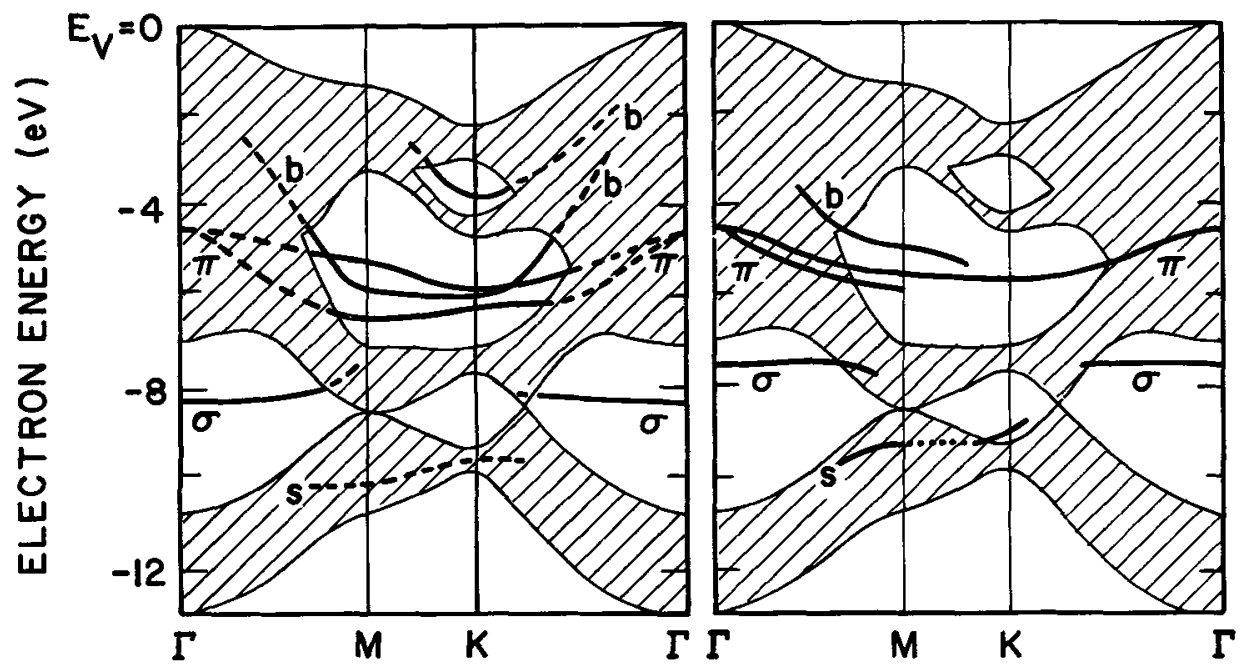

FiG 5. - Comparison of theoretically calculated (left hand panel) and experimentally measured (right hand panel) surface energy band structures for $\mathrm{Cl}$ adsorbed on $\mathrm{Si}(111)$ Cross-hatched areas represent the two-dimensional projection of the bulk Si band structure. (Larsen et $a$. . Ref. [8].) 

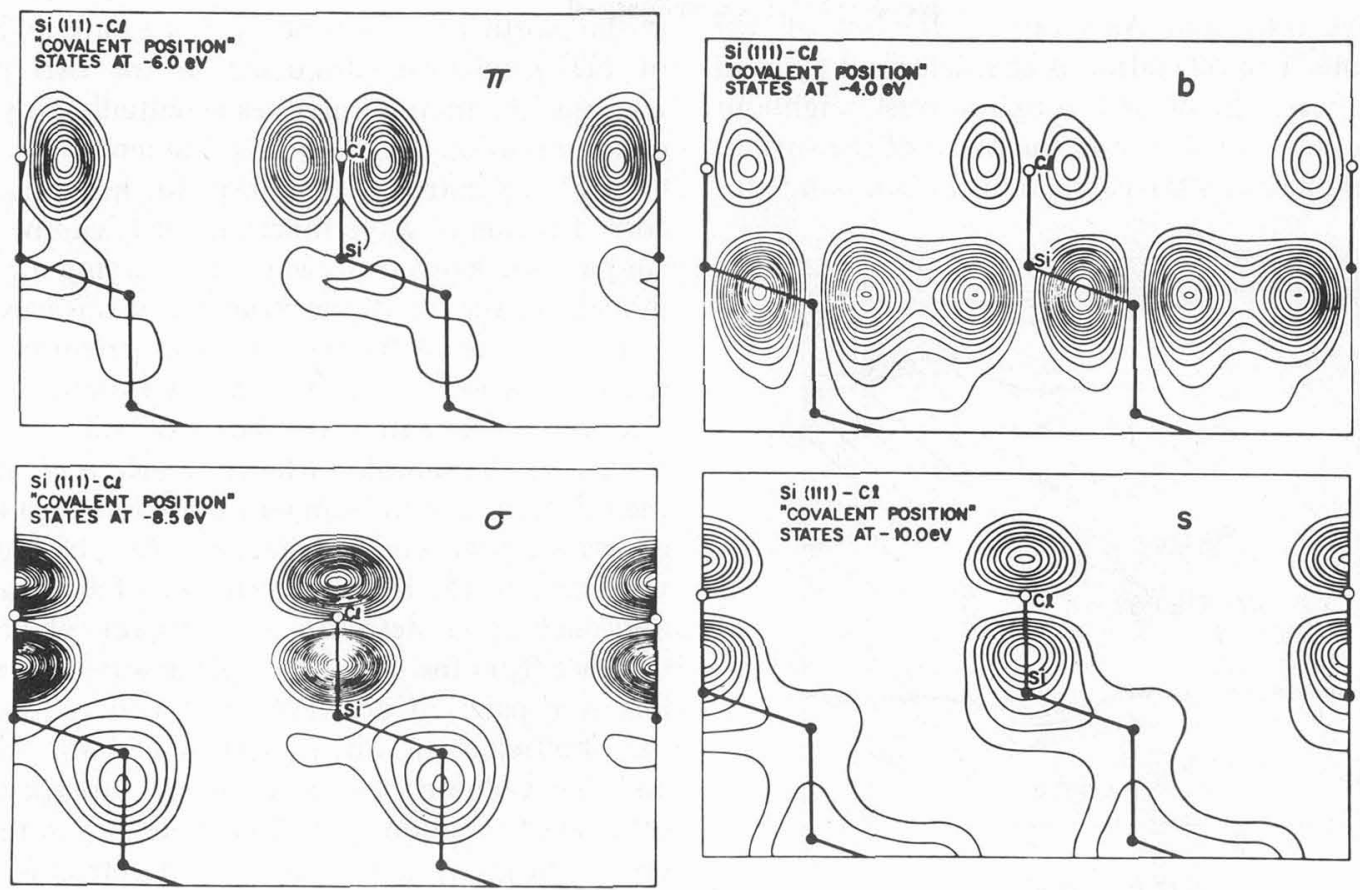

FIG. 6. - Charge-density contour maps, calculated by the pseudopotential method, for the four levels $\sigma, \pi, \mathrm{b}$ and s observed experimentally and indicated in figure 5. (Larsen et al., Ref. [8].)

The complete bonding situation however is considerably more complicated, and requires consideration of states like those designated $\mathrm{b}$ and $\mathrm{s}$. From figure 6 it is seen that the $« b$ » states involve the back-bonding or transverse orbitals between the first and second atomic layers of $\mathrm{Si}$. The « $\mathrm{s}$ » states are similar to the $\sigma$ states but involve $\mathrm{Si}$ s rather than $\mathrm{Si} \mathrm{p}_{z}$ orbital content. The final conclusion is that angle-resolved photoemission, in concert with semiempirical model calculations, offers the possibility of disentangling the full complexity of the chemisorptive bond.

4. $\boldsymbol{E}\left(\boldsymbol{k}_{\|}\right)$analysis : surface states. - The $E\left(k_{\|}\right)$ techniques described above can be applied also to the determination of the structure of clean surfaces through the examination of intrinsic surface states. As an example we discuss some recent work on the (110) face of GaAs. Earlier work of a similar nature had been done on $\mathrm{Si}$ (111) [10].

Some experimental photoelectron spectra taken on GaAs (110) by Knapp and Lapeyre [11] are shown in figure 7. These spectra display surface states, shown cross-hatched, from just below the valence band maximum, $E_{\mathrm{v}}$. In these spectra $\theta$ is kept constant, and $k_{\|}$is changed by varying the photon energy, and thereby varying $E$ in equation (2). Appreciable dispersion is observed, and Knapp and Lapeyre showed that their results were consistent with available model calculations [12] in that the surface state band has its maximum at the zone center $\bar{\Gamma}$ and its minimum at the zone boundary. There were however some discrepancies concerning the precise energy of the surface state, and the magnitude of the dispersion. A new

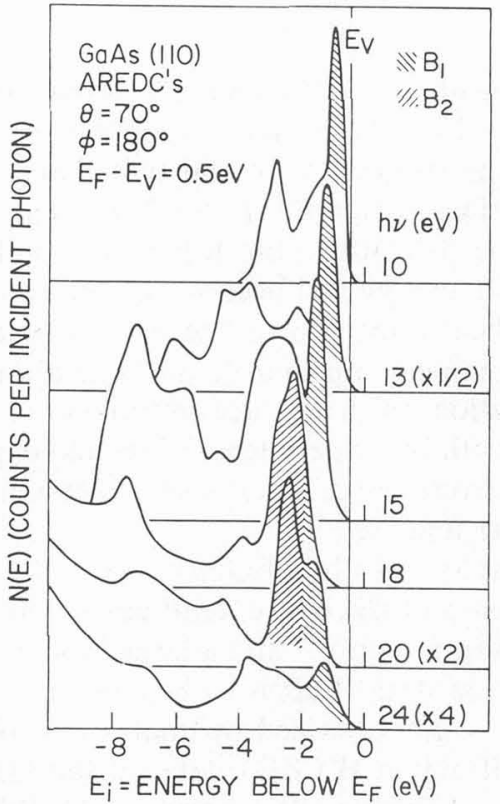

FIG. 7. - Angle-resolved photoemission spectra at various photon energies taken on $\mathrm{GaAs}(110)$ in a region where emission from intrinsic surface states, designated $\mathrm{B}_{1}$ and $\mathrm{B}_{2}$, is particularly intense. (Knapp and Lapeyre, Ref. [1].)

interpretation by Pandey et al. [13] focuses attention on the doublet nature of the surface state (indicated by different cross-hatching in figure 7) and leads to a determination of the surface structure.

The calculations of Pandey et al. employed the linear combination of atomic orbitals (LCAO) method [13]. Several different structural models were considered, including the relaxation model in which surface $\mathrm{Ga}$ 
atoms move into, and As atoms move out of, the surface plane. The relaxation is characterized by a tilt angle $\theta_{\mathrm{T}}$ between the plane through nearest neighbour surface $\mathrm{Ga}$ and As atoms and the plane of the surface itself. Figure 8 shows the calculated surface bands for

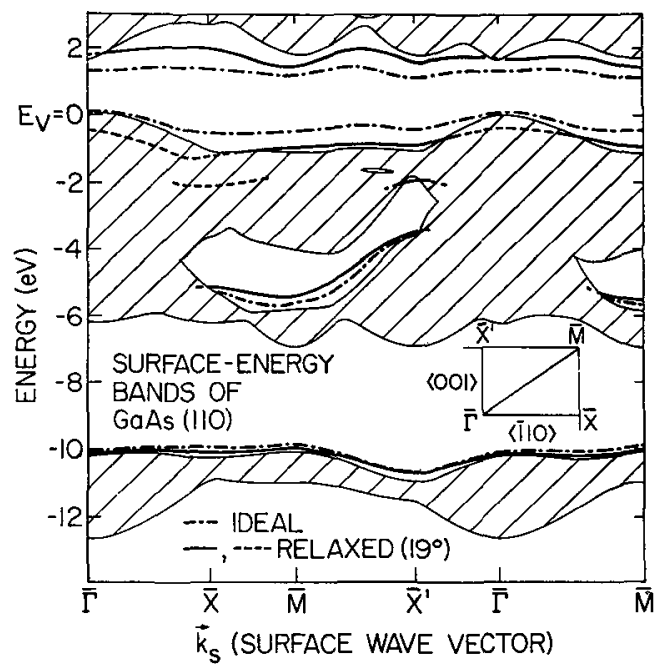

FiG. 8. - Surface energy bands of $\mathrm{GaAs}(110)$ calculated by the semiempirical LCAO method. Cross-hatched regions represent the two-dimensional projection of the bulk $\mathrm{GaAs}$ band structure. (Pandey et al. Ref. [13].)

both the ideal $\left(\theta_{\mathrm{T}}=0^{\circ}\right)$ and a relaxed surface for which $\theta_{\mathbf{T}}=19^{\circ}$. Once again, cross-hatched regions represent the projection of the bulk band structure on the surface Brillouin zone. On relaxation, the surface state just above the top of the bulk valence bands falls in energy and becomes a surface resonance lying just below the top of the valence bands. New surface resonances appear at $\bar{X}$ and $\overline{\mathrm{X}}^{\prime}$ at about $-2 \mathrm{eV}$. The prediction of a surface resonance doublet is consistent with the experimental data and explains the apparently overly large amount of dispersion reported in the earlier analyses.

The relaxed results obtained with $\theta_{\mathrm{T}}=19^{\circ}$ in figure 8 represent the best overall agreement with the spectra shown in figure 7 and a large amount of other photoemission data. This is to be compared with the value of $\theta_{\mathrm{T}}=35^{\circ}$ obtained by analysis of low energy electron diffraction (LEED) data on the GaAs (110) surface using a similar relaxation model [14]. The issue here is not which, if either, of these values is the correct one. The important point that has been established is that photoemission, particularly in its angle-resolved form, is now sufficiently incisive, and the ability to calculate surface $E\left(k_{\|}\right)$relations is sufficiently well developed, that we can contemplate using the combination for quantitative surface structure determination.

5. Parity considerations. - It will be noted that the new interpretive approaches opened up by angleresolved photoemission are characterized by extreme economy in their assumptions concerning the physics of the photoemission process. For example, in the use of $E\left(k_{\|}\right)$relations discussed in the two preceding sections, the method invokes essentially only the laws of conservation of energy and momentum. As another example of extreme economy, we now discuss how consideration of wave-function parity can be used as a simple, yet powerful, way of verifying or refuting models for the electronic structure of surfaces.

The essence of the method is to perform an angleresolved experiment in which one isolates the photoelectrons propagating in planes of mirror symmetry normal to the sample surface. In such a mirror plane, the solutions of Schrödinger's equation must be of odd or even parity under reflection. As pointed out by Hermanson [15], the wave function of a photoelectron approaching a detector in a mirror plane a long distance from the sample is a plane wave and therefore has even parity. Construction of a wave function for the photoelectron which is valid in both the sample and the vacuum can be done by superposing only other even functions; the final state $|f\rangle$ in the optical transition giving rise to the photoelectron is therefore unambiguously even. The strength of an optical transition to this state is given by the matrix element

$$
M_{\mathrm{fi}}=\langle\mathrm{i}|\mathbf{A} \cdot \boldsymbol{\nabla}| \mathrm{f}\rangle \text {. }
$$

The operator A. $\nabla$ is of even parity if the polarization vector $\mathbf{A}$ is parallel to the mirror plane $\left(\mathbf{A}_{\|}\right)$and odd if $\mathbf{A}$ perpendicular to the mirror plane $\left(A_{\perp}\right)$. A non-zero matrix element is therefore obtained with either $A_{\perp}$ and $|\mathrm{i}\rangle$ odd, or $A_{\|}$and $|\mathrm{i}\rangle$ even. (Unfortunately, these selection rules are not so clearcut in the case of degeneracies or where spin-orbit effects are significant.)

Anderson et al. [16] have recently applied these ideas to the analysis of angle-resolved data on the W (001) surface with a saturation coverage of $\mathbf{H}$. There are two normal mirror planes for this system - one containing the [100] azimuth, and the other containing the [110] azimuth. For certain $\mathrm{H}$ induced levels in the -7 to $-4 \mathrm{eV}$ range relative to the Fermi level, Andersen et al. assign even parity about [100] but odd parity about [110]. Since a $H$ 1s orbital is even, this result is, at first sight, puzzling. However Smith and Mattheiss [17], in a preliminary LCAO analysis of this system similar to those described in sections 3 and 4 , pointed out that since there are two $\mathrm{H}$ atoms for each surface $\mathrm{W}$ atom at saturation, it is necessary to consider both symmetric and antisymmetric combinations of $1 \mathrm{~s}$ orbitals. Although the details are complicated, the basic conclusion of Smith and Mattheiss was that the principal component of the chemisorptive bond for this system was coupling between the antisymmetric $H 1$ s combination and the $d_{x^{2}-y^{2}} \mathrm{~W}$ orbitals. This configuration is illustrated in figure 9 , and is seen to have parity properties in agreement with experiment. Anderson et al. further mention that there are details of the experimental results not in agreement with the model. However, 


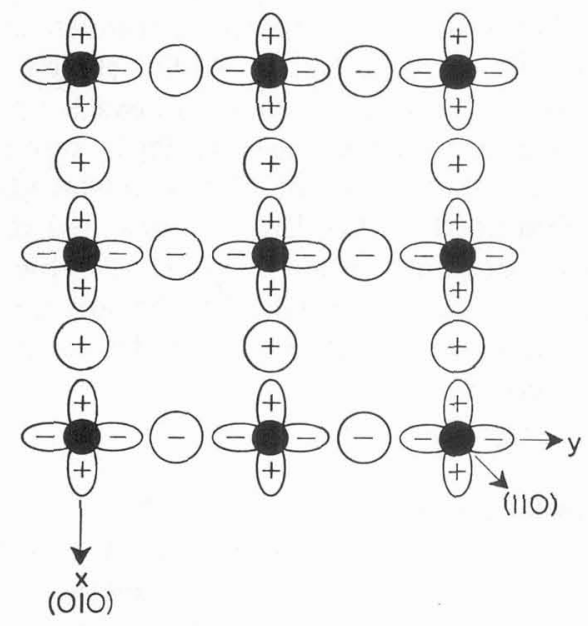

FIG. 9. - Proposed bonding configuration for the saturation adsorption of $\mathrm{H}$ on W(001). (Anderson et al., Ref. [16].)

it is difficult to explain how odd parities can be obtained at all unless one invokes the main feature of the model, antisymmetric combinations of $1 \mathrm{~s}$ orbitals.

6. Core level photoemission. - This article has focused on surface structure determination at the expense of lengthier discussions on the more traditional applications of photoemission, namely, electronic structure determination. This emphasis is justified on the grounds that such activity represents a strong current trend in both angle-integrated and angle-resolved photoemission, and that the need for routine methods for surface structure determination is a pressing one. Measuring the anisotropy of photoemission from core levels in adsorbed species has been proposed as a particularly promising method for surface structure determination, and will be discussed briefly here.

Following Liebsch [18], we write the wave function at the position $\mathbf{R}$ of the detector of a photoelectron emitted from a core level of an atom at the origin as two parts :

$$
\Psi(\mathbf{R})=\Psi^{0}(\mathbf{R})+\Psi^{1}(\mathbf{R}) .
$$

This formal separation is represented pictorially in

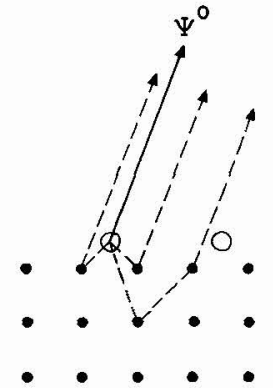

(a)

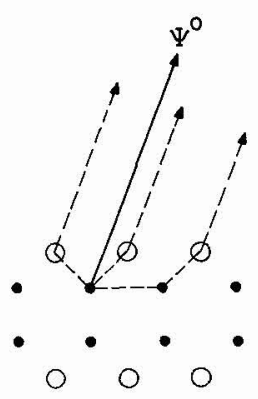

(b)
FIG. 10. - Schematic representation of the scattering geometry for core level photoemission from (a) an adsorbed Cs atom on the $\mathrm{W}(001)$ surface, (b) an In atom within the layer compound InSe. figure $10 a$. The contribution $\Psi^{0}$ is termed the direct wave and is represented as a full arrow propagating directly towards the detector. The dashed arrows represent indirect waves which have been singly - or multiply - scattered before arriving at the detector. It is the interference of these scattered waves with each other and with $\Psi^{0}$ which gives rise to the emission anisotropies. The physics is therefore formally similar to that of LEED or Extended X ray Absorption Fine Structure (EXAFS). A practical technique based on this phenomenon might appropriately be termed Photoelectron Diffraction (PhD). Such a technique has potential advantages over LEED. Because of its energy selectivity, one can tune in on core levels specific to surface atoms. Also, the adsorbed layer does not have to be periodic as in LEED.

To test the feasibility of $\mathrm{PhD}$, experiments have been performed recently on the core levels of $\mathrm{Cs}$ adsorbed on W (001). For purposes of comparison, similar experiments were performed using In core levels in the layer compound InSe. The atomic arrangement in InSe is shown in figure $10 \mathrm{~b}$. The In atoms are interior to the material, so that In core photoelectrons must traverse the surface Se layer. This therefore represents a forward-scattering configuration in contrast to the back-scattering configuration represented by Cs on W (001) and shown in figure 10a.

Radial plots for the azimuthal dependence of the core level photoemission intensity are shown in figure 11 . These results were obtained by setting the electron energy analyzer at a fixed angle $\theta$, and then monitoring the photoelectron counting rate as the sample was rotated about its surface normal [19]. The results for $\mathrm{Cs}$ on $\mathrm{W}(001)$, shown in figure $11 \mathrm{a}$,
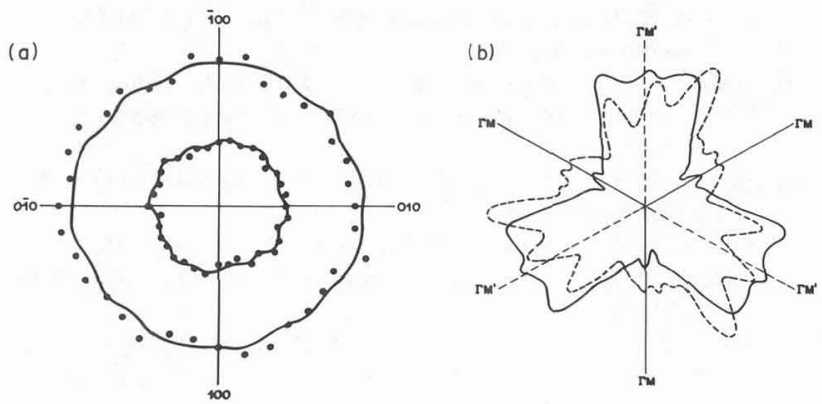

FIG. 11. - Azimuthal dependence of photoemission from core levels : (a) $5 \mathrm{p}_{3 / 2}$ level of $\mathrm{Cs}$ on $\mathrm{W}(001)$ for low (inner data, $h \omega=21 \mathrm{eV}, \theta=60^{\circ}$ ) and relatively high (outer data, $h \omega=24 \mathrm{eV}$, $\theta=60^{\circ}$ ) coverages; (b) $4 \mathrm{~d}_{5 / 2}$ level of In in the layer compounds InSe, $h \omega=33 \mathrm{eV}, \theta=60^{\circ}$. The full and dashed curves in $(b)$ correspond respectively to data taken for the emission direction in and out of the plane of incidence. (Smith et al., Ref. [19].)

display only small azimuthal anisotropy. The inner and outer curves of figure $11 a$ correspond respectively to low ( 0.04 monolayer) and relatively high ( $\sim 0.25$ monolayer) coverage of $\mathrm{Cs}$. The results for In $4 \mathrm{~d}$ emission from InSe, shown in figure $11 b$, display strong azimuthal anisotropy. (The full and 
dashed curves were obtained with analyzers in and out of the plane of incidence respectively.)

Although it is dangerous to draw sweeping conclusions from such a small amount of data, the results of figure 11 , taken at face value, suggest that, in the back-scattering configuration, $\Psi^{1}$ is too weak to bring about appreciable interference with the direct wave $\Psi^{0}$. Proponents of the oriented molecule approach of section 2 , in which back-scattering from the substrate is neglected, should derive comfort from this conclusion. It is a discouraging result, however, as far as the prospects of PhD are concerned. Theoretical prospects. are also somewhat discouraging, since analysis of data such as that in figure 11 would appear to require development of a full-blown theory of the photoemission process such as that alluded to in the intro- duction. The author hopes that his pessimism concerning $\mathrm{PhD}$ will be proved wrong. At this stage however, the worker interested in using photoemission for surface structure determination might be best advised to consider the angle-integrated surface-EXAFS method described by Lee [20], the oriented molecule approach, or the angle-resolved photoemission methods discussed above wherein the surface atomic structure is inferred indirectly via the surface electronic structure.

Acknowledgments. - I am indebted to a number of workers, and in particular J. Anderson, J. W. Davenport and K. C. Pandey, for their kindness in communicating results prior to publication.

\section{References}

[1] See for example

Pendry, J. B., J. Physique Colloq. 39 (1978) C4-142.

[2] Eastman, D. E.. in Proceedings of the IV International Conference on Vacuum Ultraviolet Radiation Physics, Hamburg, July 22-26, 1974, edited by E. Koch, R. Haensel and E. Kunz (Pergamon, New York) 1974, p. 417 ;

SPICER, W. E., ibid., p. 545.

[3] Davenport, J. W., Phys. Rev. Lett. 36 (1976) 945; thesis, University of Pennsylvania (unpublished).

[4] Allyn, C. L., Gustafsson, T. and Plummer, E. W., Chem. Phys. Lett. 47 (1977) 127. For earlier studies, see

Williams, P. M., Butcher, P., WoOd, J. and JaCOBI, K. Phys. Rev. B 14 (1976) 3215;

Smith, R. J., Anderson, J. and Lapeyre, G J., Phys Rev. Lett. 37 (1976) 1081 ;

Apal, G., Wehner, P. S., Williams, R. S., Stohr, J. and SHIRLey, D. A., Phys. Rev. Lett. 37 (1976) 1497 ;

Lloyd, D. R., Quinn, C. M. and Richardson, N. V., Solid State Commun. 20 (1976) 409.

[5] Lloyd, D. R., Faraday Discuss. Chem. Soc. 58 (1974) 136 and references therein.

[6] Gadzuk, J. W., Phys. Rev. B 10 (1974) 5030. Refinements and extensions of this basic model are being worked on; see for example

Jacobi, K., Scheffler, M., Kambe, K. and Forstmann, F., Solid State Commun. 22 (1977) 17.

[7] Smith, N. V. and Traum, M. M., Phys. Rev. B 11 (1975) 2087

Larsen, P. K., Chiang, S. and Smith, N. V., Phys. Rev. B 15 (1977) 3200 ;

Thiry, P., Petroff, Y., Pinchaux, R., Gulllot, C., Ballu, Y., LeCante, J., Paigné, J. and Levy, F., Solid State Commun. 22 (1977) 685;
Petroff, Y., J. Physique Colloq. 39 (1978) C4-149.

[8] Larsen, P. K., Smith, N. V., Schlutter, M., Ho, K. M. and COHEN, M. L., Phys. Rev. (submitted for publication).

[9] Schluter, M., Rowe, J. E., Margaritondo, G., Ho, K. M. and CoHEN, M. L., Phys. Rev. Lett. 37 (1976) 1632.

[10] Traum, M. M., Rowe, J. E. and Smith, N. V., J. Vac. Sci. Technol. 12 (1975) 298;

Ciraci, S. and Batra, I. P., Solid State Commun. 18 (1976) 1149.

[11] Knapp, J. A. and Lapeyre, G. J., J. Vac: Sci. Technol. 13 (1976) 757.

[12] Joannopoulos, J. D. and Cohen, M. L., Phys. Rev. B 10 (1974) 5075 ;

Chelrowsky, J. R. and Cohen, M. L., Phys. Rev. B 13 (1976) 826.

[13] Pandey, K. C., Freeouf, J. L. and Eastman, D. E., J. Vac. Sci. Technol. 14 (1977) 904.

[14] Lubinsky, A. R., Duke, C. B., Lee, B. W. and Mark, P., Phys. Rev. Lett. 36 (1976) 1058.

[15] Hermanson, J., Solid State Commun. 22 (1977) 9; for an earlier treatment, see

Gobeli, G. W., Alleen, F. G. and Kane, E. O., Phys. Rev. Lett. 12 (1964) 94.

[16] Anderson, J., Lapeyre, G. J. and Smith, R. J., Phys. Rev. (submitted for publication).

[17] Smith, N. V. and Mattheiss, L. F., Phys. Rev. Lett. 37 (1976) 1494

[18] Liebsch, A., Phys. Rev. B 13 (1976) 544.

[19] Smith, N V., Larsen, P. K. and Chiang, S., Phys. Rev. B 16 (1977) 2699.

[20] LeE, P. A., J. Physique Colloq. 39 (1978) C4-120. 